\title{
Correction to: In Vitro to In Vivo Extrapolation of Metabolic Clearance for UGT Substrates Using Short-Term Suspension and Long-Term Co-cultured Human Hepatocytes
}

\author{
Luca Docci, ${ }^{1,2}$ Florian Klammers, ${ }^{1}$ Aynur Ekiciler, ${ }^{1}$ Birgit Molitor, ${ }^{1}$ Kenichi Umehara, ${ }^{1}$ Isabelle Walter, ${ }^{1}$ \\ Stephan Krähenbühl, ${ }^{2}$ Neil Parrott, ${ }^{1}$ and Stephen Fowler ${ }^{1,3}$
}

Published online 6 November 2020

Correction to: The AAPS Journal volume 22, issue 6 Article number: 131 (2020) https://doi.org/10.1208/s12248-020-00482-9

During production, the figure captions for Fig. 1 and Fig. 2 were inadvertently switched in the proofing stage. The correct figure captions are below and the original article has been corrected.

Fig. 1 Distribution of body-weight normalized, observed plasma clearance for 13 UGT substrates either after intravenous (circles) or oral (squares; divided by bioavailability) administration. The circle/square size indicates the study sample size with smallest ( $n \leq 5$ subjects) to largest size ( $n \geq 31$ subjects). The error bar represents the standard deviation

Fig. 2 In vitro intrinsic clearance values for 13 UGT substrates generated in three separate experiments in a suspended hepatocytes and $\mathbf{b}$ HepatoPac. Shown are the individual measurements (circles), the mean values (horizontal lines), and standard deviation (error bars)

Publisher's Note Springer Nature remains neutral with regard to jurisdictional claims in published maps and institutional affiliations.

The online version of the original article can be found at https://doi.org/ 10.1208/s12248-020-00482-9

\footnotetext{
${ }^{1}$ Pharmaceutical Sciences, Roche Innovation Center Basel, F.Hoffmann-La Roche Ltd. Grenzacherstrasse 124, 4070, Basel, Switzerland.

${ }^{2}$ Department of Biomedicine, University of Basel, Hebelstrasse 20, 4031, Basel, Switzerland.

${ }^{3}$ To whom correspondence should be addressed. (e-mail: stephen.fowler@roche.com)
} 